\title{
Arterial Stiffness is Associated With Diabetic Retinopathy in Korean Type 2 Diabetic Patients
}

\author{
Yong-Woon Yun', Min-Ho Shin'1, Young-Hoon Lee ${ }^{2}$, Jung-Ae Rhee', Jin-Su Choi ${ }^{1}$ \\ 'Department of Preventive Medicine, Chonnam National University Medical School, Gwangju; \\ ${ }^{2}$ Department of Preventive Medicine, Wonkwang University Medical School, Iksan, Korea
}

\begin{abstract}
Objectives: We evaluated the association between common carotid artery intima-media thickness (CCA-IMT), brachialankle pulse wave velocity (baPWV), carotid plaque, and peripheral arterial disease (PAD) as indicators of macroangiopathy and diabetic retinopathy as an indicator of microangiopathy in type 2 diabetic patients.

Methods: We analyzed 605 type 2 diabetic patients registered at a public health center in Korea. Following overnight fasting, venous blood and urine samples were collected and analyzed. The CCA-IMT, levels of carotid plaque, baPWV, and ankle-brachial index ( $\mathrm{ABI})$ of the subjects were assessed. We used non-mydriatic fundus photography to diagnose diabetic retinopathy. Multiple logistic regression analyses were used to evaluate the association between macroangiopathy and diabetic retinopathy. CCA-IMT and baPWV were divided into tertiles: CCA-IMT, 0.39 to $0.65 \mathrm{~mm}$, 0.66 to $0.78 \mathrm{~mm}$, and 0.79 to $1.30 \mathrm{~mm}$; baPWV, 9.9 to $15.8 \mathrm{~m} / \mathrm{s}, 15.9$ to $18.9 \mathrm{~m} / \mathrm{s}$, and 19.0 to $38.0 \mathrm{~m} / \mathrm{s}$.

Results: The association between baPWV and diabetic retinopathy remained significant after adjustment, with an increasing odds ratio (OR) in the second tertile (OR, 2.41; 95\% confidence interval [Cl], 1.27 to 4.55$)$ and the third tertile $(\mathrm{OR}, 4.63$; $95 \% \mathrm{Cl}, 2.33$ to 9.21). No significant differences were observed in carotid plaque, PAD, and each tertile of CCA-IMT.

Conclusions: BaPWV was associated with diabetic retinopathy, while CCA-IMT, carotid plaque, and PAD were not. This study suggests that the association between macroangiopathy and microangiopathy may be attributable to functional processes rather than structural processes within the vascular system.

Key words: Carotid plaque, Diabetes mellitus, Diabetic retinopathy, Intima-media thickness, Peripheral arterial disease, Pulse wave velocity J Prev Med Public Health 2011;44(6):260-266
\end{abstract}

\section{INTRODUCTION}

Recent epidemiologic studies have shown a significant rise in the prevalence of type 2 diabetes worldwide, resulting in increased burdens on individuals and health care systems $[1,2]$. This disease creates huge social, economic, and health problems, caused mainly by diabetic macro- and microvascular complications [2,3] The frequent coexistence of diabetic macro- and microangiopathy was described decades ago [4-6].

For macroangiopathy, several measures of subclinical atherosclerosis have been developed as surrogate measures of cardiovascular disease (CVD) [7-11]. Atherosclerosis consists of two distinct processes: a structural process (atherosis) and a functional process (sclerosis) [12]. Common carotid artery intima-media thickness (CCA-IMT), ankle-brachial index (ABI), and carotid plaque presence are considered parameters of structural changes in atherosclerosis whereas brachialankle pulse wave velocity (baPWV) is a convenient

(c) This is an Open Access article distributed under the terms of the Creative Common Attribution Non-Commercial License (http://creativecommons.org/licenses/by-nc/3.0/) which permits unrestricted non-commercial use, distribution, and reproduction in any medium, provided the original work is properly cited. measure of functional changes in atherosclerosis [13]. Similar to subclinical atherosclerosis, diabetic microangiopathy is associated with CVD risk in type 2 diabetic patients [14-16].

Such evidence is supported by a "common mechanism" for the development of macro- and microangiopathy in type 2 diabetes [17-20]. Several studies have evaluated the association between macroangiopathy and diabetic retinopathy [20-24]. However, these associations have not been adequately explored, particularly in the Korean population.

In this study, we evaluated the association between CCA-IMT, baPWV, carotid plaque, and peripheral arterial disease (PAD) as indicators of macroangiopathy, and diabetic retinopathy as an indicator of microangiopathy in type 2 diabetic patients. 


\section{METHODS}

\section{Subjects}

The study population consisted of 1275 type 2 diabetic patients registered at a public health center of Seo-gu, Gwangju, and Gokseong-gun, Jeollanamdo, Korea. The subjects were participants in the surveys of the Seo-gu study (conducted between November 2008 and December 2008), and the Gokseong-gun study (conducted between June 2009 and November 2009).

In total, 1275 eligible subjects (Seo-gu: 681, Gokseong-gun: 594) were invited by telephone to participate. Of these, 709 (Seo-gu: 327, Gokseong-gun: 382) underwent clinical examinations following interviews. The response rate for the study was $55 \%$ (Seo-gu: $48.0 \%$, Gokseong-gun: 64.3\%). Ten patients who did not provide blood samples and twenty-six who did not provide urine samples were excluded from the study. Sixty-eight patients had no HbA1c, CCA-IMT, carotid plaque, baPWV, history of diabetic duration, or fundoscopic outcome and were excluded from the analysis. After excluding these patients, a total of 605 patients (Seo-gu: 290, Gokseong-gun: 315) participated in the study.

The study protocol was approved by the Institutional Review Board of Chonnam National University Hospital, and informed consent was obtained from each subject.

\section{Methods}

A well-trained examiner interviewed the patients using a questionnaire that included items on smoking status, diabetic duration, anti-diabetic medication use, antihypertension medication use, and a history of past illnesses.

Anthropometric measurements, including weight, height, and waist measurements, were obtained using standard techniques. The body mass index (BMI) was calculated using the following formula: weight $(\mathrm{kg}) /$ height $^{2}\left(\mathrm{~m}^{2}\right)$. Blood pressure was measured on the right upper arm using a mercury sphygmomanometer (Baumanometer; WA Baum Co., Inc., Copiague, NY, USA) with an appropriately sized cuff after subjects had rested at least $10 \mathrm{~min}$ in a sitting position. The first appearance (phase I) and disappearance (phase V) of Korotkoff sounds were used to define systolic and diastolic blood pressure, which was read to the nearest 2 $\mathrm{mmHg}$. Two consecutive measurements of systolic and diastolic blood pressure were taken in 5 minutes intervals, and the average was used in the analysis.

All laboratory specimens were taken following overnight fasting. The serum was separated onsite and stored at $-70^{\circ} \mathrm{C}$ until further analysis. All serum samples were examined using an automatic analyzer (Hitachi7600, Hitachi Ltd, Tokyo, Japan). HbA1c was measured by high-performance liquid chromatography (HPLC) using the Variant II (Bio-Rad, Hercules, CA, USA). Total cholesterol, high-density lipoprotein (HDL) cholesterol, and triglyceride concentrations were analyzed using enzymatic methods. Low-density lipoprotein (LDL) cholesterol was estimated as proposed by Friedewald et al. [25], except when triglyceride levels exceeded $400 \mathrm{mg} / \mathrm{dL}$. In such instances, the data were treated as missing.

Kidney function was assessed using the estimated glomerular filtration rate (eGFR), which was calculated by the Modification of Diet in Renal Disease (MDRD) formula as follows [26]: $186.3 \times$ (serum creatinine$1.154) \times($ age -0.203$) \times 0.742$ (if female), with the serum creatinine concentration expressed in $\mathrm{mg} / \mathrm{dL}$.

Non-mydriatic digital images of the retina of each eye were taken using a retinal camera (TRC-NW200, Topcon Co., Japan). Retinopathy was considered present if any characteristic lesions as defined by the Early Treatment Diabetic Retinopathy Study (ETDRS) severity scale were present (microaneurysms, hemorrhages, cotton wool spots, intraretinal microvascular abnormalities, hard exudates, and new retinal vessels). If the ETDRS severity score was 10 , the patient was defined as having diabetic retinopathy [27].

The CCA-IMT and the presence of carotid plaques were assessed by physicians using high-resolution mode B ultrasonographic scans of the carotid arteries (SonoAce 9900, Medison, Korea) with an electrical linear array transducer $(7.5 \mathrm{MHz})$. The CCA-IMT was defined as the distance from the leading edge of the first echogenic line to the second echogenic line, which indicated the media-adventitia interface. Images of the thickest point within $10 \mathrm{~mm}$ from the carotid bulb to the CCA were saved as CCA-IMT, and then measured using SigmaScan Pro version 5.0.0 (Systat Software Inc., Chicago, IL, USA).

The examiners evaluated the CCA, carotid bulb, and internal carotid artery to determine the levels of carotid plaque. Protrusions into the lumen that were $100 \%$ thicker than the nearest area were defined as plaques. If the plaque was the thickest point, the distance to the nearest point without plaques was defined as the CCAIMT. The presence of carotid plaques was recorded if at 
Table 1. General characteristics of the study group with and without retinopathy

\begin{tabular}{|c|c|c|c|}
\hline Variables & Without retinopathy $(n=491)$ & With retinopathy $(n=114)$ & $p$-value ${ }^{2}$ \\
\hline Men (\%) & $144(29.3)$ & $39(34.2)$ & 0.307 \\
\hline Age (y) & $68.7 \pm 9.5$ & $64.4 \pm 10.9$ & $<0.001$ \\
\hline Duration of diabetes (y) & $7.7 \pm 7.2$ & $12.9 \pm 9.4$ & $<0.001$ \\
\hline Height $(\mathrm{cm})$ & $154.2 \pm 8.5$ & $155.4 \pm 8.1$ & 0.295 \\
\hline Weight (kg) & $58.8 \pm 10.1$ & $57.6 \pm 9.7$ & 0.238 \\
\hline Body mass index $\left(\mathrm{kg} / \mathrm{m}^{2}\right)$ & $24.7 \pm 3.8$ & $23.8 \pm 3.5$ & 0.035 \\
\hline Total cholesterol (mg/dL) & $193.7 \pm 43.1$ & $196.4 \pm 48.7$ & 0.558 \\
\hline Triglycerides (mg/dL) ${ }^{1}$ & $153.0(103.2-228.2)$ & $145.7(99.9-203.4)$ & $0.320^{3}$ \\
\hline HDL cholesterol (mg/dL) & $47.3 \pm 11.1$ & $47.7 \pm 12.7$ & 0.729 \\
\hline $\mathrm{HbA1c}(\%)$ & $7.3 \pm 1.5$ & $8.2 \pm 1.8$ & $<0.001$ \\
\hline eGFR $\left(\mathrm{mL} / \mathrm{min} / 1.73 \mathrm{~m}^{2}\right)$ & $65.3 \pm 15.6$ & $65.1 \pm 17.8$ & 0.909 \\
\hline Hypertension (\%) & $258(52.5)$ & $54(47.4)$ & 0.319 \\
\hline PAD (\%) & $52(10.6)$ & $10(8.8)$ & 0.564 \\
\hline CCA-IMT (mm) & $0.76 \pm 0.42$ & $0.71 \pm 0.14$ & 0.216 \\
\hline Carotid plaques (\%) & $236(48.1)$ & $60(52.6)$ & 0.380 \\
\hline baPWV $(\mathrm{m} / \mathrm{s})^{1}$ & $17.1(14.9-19.9)$ & $17.7(15.8-20.5)$ & $0.072^{3}$ \\
\hline
\end{tabular}

HDL: high-density lipoprotein, eGFR: estimated glomerular filtration rate, PAD: peripheral arterial disease, CCA-IMT: common carotid artery intimamedia thickness, baPWV: brachial-ankle pulse wave velocity.

All values are given as $n(\%)$ or mean \pm standard deviation.

${ }^{1}$ Values are log transformed and given as median(interquartile range), ${ }^{2} p$-value, as determined by the t-test or chi-square test as appropriate, ${ }^{3} p$-value, as determined by the Mann-Whitney $U$ test.

least one lesion was detected in six segments.

After a minimum 5 minutes rest period, the baPWV and ABI were calculated automatically in the supine position using the VP-1000 system (Colin Co., Komaki, Japan) with cuffs around both arms and ankles. If any of the ABIs were $<0.9$, the patient was defined as having PAD.

\section{Statistical Analyses}

Data are provided as the mean \pm standard deviation (SD) for continuous variables, the percentage for categorical variables, and the median (interquartile range) for triglyceride and baPWV. An independent sample t-test or Mann-Whitney U test was used to examine significant differences in the mean values between the two groups, and a chi-square test was used to examine differences in the frequency. The associations between CCA-IMT, baPWV, and clinical characteristics were examined using partial correlation, controlling for age and sex. To evaluate the association between the surrogate of macroangiopathy (CCA-IMT, baPWV, carotid plaque, and PAD) and diabetic retinopathy, different models were constructed using multiple logistic regression analyses by adjusting for confounding risk variables (age, sex, duration of diabetes, HbA1c, total cholesterol (TC), log-transformed triglycerides, HDL cholesterol, eGFR, BMI, and history of hypertension). These confounding risk variables were selected through a literature review [22-24]. Because there is no clear cut-point for categorization, CCA-IMT and baPWV were divided into tertiles: CCA-IMT, 0.39 to $0.65 \mathrm{~mm}, 0.66$ to $0.78 \mathrm{~mm}$, and 0.79 to $1.30 \mathrm{~mm}$; baPWV, 9.9 to $15.8 \mathrm{~m} / \mathrm{s}, 15.9$ to $18.9 \mathrm{~m} / \mathrm{s}$, and 19.0 to $38.0 \mathrm{~m} / \mathrm{s}$.

Statistical analyses were performed with the SPSS version 19.0 (SPSS Inc, Chicago, IL, USA). A level of $\mathrm{p}$ $<0.05$ was accepted as statistically significant.

\section{RESULTS}

Table 1 summarizes the general characteristics of the study groups with and without retinopathy. The presence of diabetic retinopathy was confirmed in 114 (18.8\%) of the 605 patients who were eligible to take part in the study. The mean age of the subjects with diabetic retinopathy was significantly lower compared to subjects without diabetic retinopathy. In addition, the response rate of the Gokseong-gun study (64.3\%) was higher than that of the Seo-gu study (48.0\%), and a significant mean age difference existed between the two regions. However, the mean age of the subjects with diabetic retinopathy was significantly lower compared to subjects without diabetic retinopathy in each of the two regions. The stratified analysis also showed that there was no evidence of effect modification by region (data not shown). The mean values of HbA1c and the duration of diabetes were significantly higher among subjects with diabetic retinopathy compared to subjects without diabetic retinopathy. The baPWV was higher in subjects with diabetic retinopathy, but this was not statistically 
Table 2. Association between CCA-IMT, baPWV and clinical characteristics

\begin{tabular}{lccccr}
\hline \multirow{2}{*}{ Variables } & \multicolumn{2}{c}{ CCA-IMT } & & \multicolumn{2}{c}{ baPWV } \\
\cline { 2 - 3 } \cline { 5 - 6 } & $\begin{array}{l}\text { Correlation } \\
\text { coefficient }\end{array}$ & $p$-value & & $\begin{array}{l}\text { Correlation } \\
\text { coefficient }^{1}\end{array}$ & $p$-value \\
\hline Duration of diabetes & -0.059 & 0.147 & & 0.018 & 0.663 \\
\hline Body mass index & -0.005 & 0.912 & & -0.158 & $<0.001$ \\
\hline Total cholesterol & 0.020 & 0.618 & & 0.074 & 0.068 \\
Triglycerides & -0.013 & 0.750 & & 0.131 & 0.001 \\
HDL cholesterol & -0.052 & 0.202 & & 0.007 & 0.871 \\
HbA1c & -0.071 & 0.080 & & 0.059 & 0.146 \\
\hline eGFR & -0.009 & 0.827 & & -0.024 & 0.557 \\
\hline
\end{tabular}

CCA-IMT: common carotid artery intima-media thickness, baPWV: brachial-ankle pulse wave velocity, HDL: high density lipoprotein, eGFR: estimated glomerular filtration rate. ${ }^{1}$ partial correlation coefficient adjusted for sex and age.

significant.

The associations between CCA-IMT, baPWV, and clinical characteristics are presented in Table 2. The CCA-IMT was not correlated with clinical characteristics, while baPWV was significantly correlated with BMI $(r=-0.158, p<0.001)$ and triglycerides $(r=0.131$, $\mathrm{p}=0.001)$.

The odds ratios (ORs) for diabetic retinopathy according to the CCA-IMT levels are listed in Table 3. When adjusted for other covariates (i.e., sex, age, duration of diabetes, HbA1c, TC, log-transformed triglycerides (logTG), HDL, eGFR, BMI, and history of HTN), the CCA-IMT was not significantly associated with diabetic retinopathy.

The ORs for diabetic retinopathy according to baPWV levels are listed in Table 3. Compared to the first tertile, crude ORs (95\% confidence interval [CI]) for retinopathy were 1.45 (0.85 to 2.47) for the second tertile and 1.93 (1.16 to 3.24) for the third tertile. Adjustment for age and sex increased ORs $(95 \% \mathrm{CI})$ for retinopathy: 2.48 (1.37 to 4.49 ) for the second tertile and 4.44 (2.38 to 8.31 ) for the third tertile. Further adjustment for other covariates
(Model 2) did not change the association.

The ORs for diabetic retinopathy according to carotid plaques and PAD are listed in Table 3. When adjusted for all covariates (Model 2), the carotid plaques and PAD were not significantly associated with diabetic retinopathy.

\section{DISCUSSION}

The purpose of this study was to determine the association between macroangiopathy (CCA-IMT, baPWV, carotid plaque, and PAD) and diabetic retinopathy. We found that baPWV is significantly associated with diabetic retinopathy, whereas CCA-IMT, carotid plaque, and PAD are not.

There was no significant association between CCAIMT and diabetic retinopathy among type 2 diabetic patients after adjusting for cardiovascular risk factors. These results are consistent with the findings of the Cardiovascular Health Study (CHS) [21]. However, the Chennai Urban Rural Epidemiology Study (CURES-2) and the Atherosclerosis Risk In Communities (ARIC) study reported a positive relationship between CCAIMT and diabetic retinopathy $[22,28]$. We did not observe an independent association between carotid plaque, PAD, and diabetic retinopathy, whereas a positive relationship has previously been observed $[20,29]$. However, carotid plaque and PAD have been shown not to be significantly associated with diabetic retinopathy in some studies [30-32]. Selective survival may provide a potential explanation for these discrepant results. Severe atherosclerosis and retinopathy in diabetic patients were both associated with an increased risk of death, which may have resulted in individuals with these conditions being less likely to be examined

Table 3. Odds ratios for diabetic retinopathy according to CCA-IMT level, baPWV level, carotid plaque, PAD

\begin{tabular}{|c|c|c|c|c|c|}
\hline Variables & Range & $\begin{array}{c}\text { Crude OR } \\
(95 \% \mathrm{Cl})\end{array}$ & $\begin{array}{c}\text { Model } 1^{1} \mathrm{OR} \\
(95 \% \mathrm{Cl})\end{array}$ & $\begin{array}{l}\text { Model } 2^{2} \text { OR } \\
(95 \% \mathrm{Cl})\end{array}$ & $\begin{array}{l}p \text {-value for trend } \\
\text { in Model } 2\end{array}$ \\
\hline \multicolumn{6}{|l|}{ CCA-IMT (mm) } \\
\hline Tertile 1 & $0.39,0.65$ & 1.00 & 1.00 & 1.00 & 0.844 \\
\hline Tertile 2 & $0.66,0.78$ & $0.86(0.53,1.40)$ & $1.12(0.67,1.86)$ & $1.16(0.67,2.02)$ & \\
\hline Tertile 3 & $0.79,1.30$ & $0.65(0.39,1.08)$ & $0.91(0.53,1.58)$ & $1.06(0.59,1.90)$ & \\
\hline \multicolumn{6}{|l|}{ baPWV (m/s) } \\
\hline Tertile 1 & $9.9,15.8$ & 1.00 & 1.00 & 1.00 & $<0.001$ \\
\hline Tertile 2 & $15.9,18.9$ & $1.45(0.85,2.47)$ & $2.48(1.37,4.49)$ & $2.41(1.27,4.55)$ & \\
\hline Tertile 3 & $19.0,38.0$ & $1.93(1.16,3.24)$ & $4.44(2.38,8.31)$ & $4.63(2.33,9.21)$ & \\
\hline Carotid plaque & No vs yes & $1.20(0.80,1.80)$ & $1.43(0.93,2.20)$ & $1.20(0.75,1.91)$ & \\
\hline PAD & No vs yes & $0.81(0.40,1.65)$ & $1.01(0.49,2.10)$ & $0.771(0.34,1.73)$ & \\
\hline
\end{tabular}

CCA-IMT: common carotid artery intima-media thickness, baPWV: brachial-ankle pulse wave velocity, PAD: peripheral arterial disease, OR: odds ratio, Cl: confidence interval.

${ }^{1}$ Adjusted for age and sex, ${ }^{2}$ Adjusted for age, sex, duration of diabetes, HbA1c, TC, log-transformed triglycerides, HDL cholesterol, eGFR, BMI, and history of hypertension. 
than patients without these conditions [33]. The other potential reasons for the discrepant results may include different sample sizes, population characteristics, the lower magnification of our images, and the age distribution of the study population.

Arterial stiffness is a major contributor to CVD [34], and baPWV is correlated with the degree of atherosclerotic vascular damage $[13,35]$. The present study showed that baPWV has a distinct association with diabetic retinopathy contrary to other surrogates. The exact mechanisms underlying the association between atherosclerosis and diabetic retinopathy are poorly understood. In previous studies, analyses were conducted separately according to each surrogate [20,22$24,29,30]$. An integrated theory to explain these observed phenomena has not been established. One possible explanation is that atherosclerosis and diabetic retinopathy share common risk factors in the causal pathway $[17,18,20,22]$. Although the adjustment for cardiovascular risk factors in our study did not change the association between surrogates of macroangiopathy and diabetic retinopathy, the possibility of an effect of unmeasured variables remained. In the present study, functional markers such as baPWV were associated with diabetic retinopathy, but structural markers such as IMT, carotid plaque, and PAD were not. This suggests that the association between macroangiopathy and microangiopathy may be due to a functional rather than structural process within the vascular system.

Interestingly, in the present study, without adjustment, there was no significant difference in the baPWV between subjects with diabetic retinopathy and subjects without diabetic retinopathy ( $\mathrm{p}$ value $=0.072$ ). However, after adjustment for age and sex, baPWV was significantly associated with diabetic retinopathy. Therefore, this phenomenon may result from the positive confounding effect of age and sex.

This study has several limitations. First, this was a cross-sectional study. The association between macroangiopathy and diabetic retinopathy cannot be taken as a causal relationship. Nonetheless, we believe that the results may help shed light on the pathophysiological connections between the development and progression of macro- and microangiopathy in diabetes. Second, the study targets type 2 diabetic patients who were registered at a public health center in a certain area and does not represent the general population across the nation. Third, the mean age of the subjects with diabetic retinopathy was significantly lower than that of subjects without diabetic retinopathy. This phenomenon may be explained by selection bias due to either selective survival or nonparticipation (restricted movement). However, we cannot conclude that this phenomenon resulted from selection bias, because we did not compare general characteristics of study participants and nonparticipants. Fourth, we conducted multiple comparisons among the four phenotypes of macroangiopathy. However, after posthoc multiple comparisons using the Bonferroni correction, these associations did not change substantially. Fifth, we used non-mydriatic color digital images of the retina. The accuracy of this method is slightly lower than the use of mydriatic color images. Despite these limitations, this study provides a valuable contribution, in that we evaluated the association between multiple phenotypes of macroangiopathy, including CCA-IMT, carotid plaque, PAD, and baPWV, and diabetic retinopathy in a single population.

In summary, baPWV is associated with diabetic retinopathy in type 2 diabetic patients, but CCA-IMT, carotid plaque, and PAD are not. Additional studies are required to further evaluate the common pathogenic mechanisms underlying these associations in Korean type 2 diabetic patients.

\section{CONFLICT OF INTEREST}

The authors have no conflicts of interest with the material presented in this paper.

\section{REFERENCES}

1. Girach A, Vignati L. Diabetic microvascular complications--can the presence of one predict the development of another? J Diabetes Complications 2006; 20(4): 228-237.

2. Malecki MT, Osmenda G, Walus-Miarka M, Skupien J, Cyganek K, Mirkiewicz-Sieradzka B, et al. Retinopathy in type 2 diabetes mellitus is associated with increased intimamedia thickness and endothelial dysfunction. Eur J Clin Invest 2008; 38(12): 925-930.

3. Jönsson B; CODE-2 Advisory Board. Revealing the cost of Type II diabetes in Europe. Diabetologia 2002; 45(7): S5S12.

4. Hiller R, Sperduto RD, Podgor MJ, Ferris FL 3rd, Wilson PW. Diabetic retinopathy and cardiovascular disease in type II diabetics. The Framingham Heart Study and the Framingham Eye Study. Am J Epidemiol 1988; 128(2): 402409.

5. Adler AI, Stratton IM, Neil HA, Yudkin JS, Matthews DR, Cull CA, et al. Association of systolic blood pressure with macrovascular and microvascular complications of type 2 
diabetes (UKPDS 36): prospective observational study. BMJ 2000; 321(7258): 412-419.

6. Stratton IM, Adler AI, Neil HA, Matthews DR, Manley SE, Cull CA, et al. Association of glycaemia with macrovascular and microvascular complications of type 2 diabetes (UKPDS 35): prospective observational study. BMJ 2000; 321(7258): 405-412.

7. Wofford JL, Kahl FR, Howard GR, McKinney WM, Toole JF, Crouse JR 3rd. Relation of extent of extracranial carotid artery atherosclerosis as measured by B-mode ultrasound to the extent of coronary atherosclerosis. Arterioscler Thromb 1991; 11(6): 1786-1794.

8. Bernard S, Serusclat A, Targe F, Charriére S, Roth O, Beaune $\mathrm{J}$, et al. Incremental predictive value of carotid ultrasonography in the assessment of coronary risk in a cohort of asymptomatic type 2 diabetic subjects. Diabetes Care 2005; 28(5): 1158-1162.

9. Lorenz MW, Markus HS, Bots ML, Rosvall M, Sitzer M. Prediction of clinical cardiovascular events with carotid intima-media thickness: a systematic review and metaanalysis. Circulation 2007; 115(4): 459-467.

10. Prati P, Tosetto A, Vanuzzo D, Bader G, Casaroli M, Canciani L, et al. Carotid intima media thickness and plaques can predict the occurrence of ischemic cerebrovascular events. Stroke 2008; 39(9): 2470-2476.

11. Vigili de Kreutzenberg S, Tiengo A, Avogaro A. Cerebrovascular disease in diabetes mellitus: the role of carotid intima-media thickness. Nutr Metab Cardiovasc Dis 2009; 19(9): 667-673.

12. Blankenhorn DH, Kramsch DM. Reversal of atherosis and sclerosis. The two components of atherosclerosis. Circulation 1989; 79(1): 1-7.

13. Zureik M, Temmar M, Adamopoulos C, Bureau JM, Courbon D, Thomas F, et al. Carotid plaques, but not common carotid intima-media thickness, are independently associated with aortic stiffness. J Hypertens 2002; 20(1): 85-93.

14. Cheung N, Wang JJ, Klein R, Couper DJ, Sharrett AR, Wong TY. Diabetic retinopathy and the risk of coronary heart disease: the Atherosclerosis Risk in Communities Study. Diabetes Care 2007; 30(7): 1742-1746.

15. Juutilainen A, Lehto S, Ronnemaa T, Pyörälä K, Laakso M. Retinopathy predicts cardiovascular mortality in type 2 diabetic men and women. Diabetes Care 2007; 30(2): 292299.

16. Romundstad S, Holmen J, Kvenild K, Hallan H, Ellekjaer H. Microalbuminuria and all-cause mortality in 2,089 apparently healthy individuals: a 4.4-year follow-up study. The Nord-Trondelag Health Study (HUNT), Norway. Am J Kidney Dis 2003; 42(3): 466-473.

17. Goldin A, Beckman JA, Schmidt AM, Creager MA. Advanced glycation end products: sparking the development of diabetic vascular injury. Circulation 2006; 114(6): 597-605.

18. Brownlee M. The pathobiology of diabetic complications: a unifying mechanism. Diabetes 2005; 54(6): 1615-1625.

19. Stern MP. Diabetes and cardiovascular disease. The “common soil" hypothesis. Diabetes 1995; 44(4): 369-374.

20. de Kreutzenberg SV, Coracina A, Volpi A, Fadini GP, Frigo AC, Guarneri G, et al. Microangiopathy is independently associated with presence, severity and composition of carotid atherosclerosis in type 2 diabetes. Nutr Metab Cardiovasc Dis 2011; 21(4): 286-293.

21. Klein R, Marino EK, Kuller LH, Polak JF, Tracy RP, Gottdiener JS, et al. The relation of atherosclerotic cardiovascular disease to retinopathy in people with diabetes in the Cardiovascular Health Study. $\mathrm{Br} J$ Ophthalmol 2002; 86(1): 84-90.

22. Rema M, Mohan V, Deepa R, Ravikumar R; Chennal Urban Rural Epidemiology Study-2. Association of carotid intima-media thickness and arterial stiffness with diabetic retinopathy: the Chennai Urban Rural Epide-miology Study (CURES-2). Diabetes Care 2004; 27(8): 1962-1967.

23. Ogawa O, Hayashi C, Nakaniwa T, Tanaka Y, Kawamori $\mathrm{R}$. Arterial stiffness is associated with diabetic retinopathy in type 2 diabetes. Diabetes Res Clin Pract 2005; 68(2): 162-166.

24. Ogawa O, Hiraoka K, Watanabe T, Kinoshita J, Kawasumi $\mathrm{M}$, Yoshii H, et al. Diabetic retinopathy is associated with pulse wave velocity, not with the augmentation index of pulse waveform. Cardiovasc Diabetol 2008; 7: 11.

25. Friedewald WT, Levy RI, Fredrickson DS. Estimation of the concentration of low-density lipoprotein cholesterol in plasma, without use of the preparative ultracentrifuge. Clin Chem 1972; 18(6): 499-502.

26. Levey AS, Bosch JP, Lewis JB, Greene T, Rogers N, Roth D. A more accurate method to estimate glomerular filtration rate from serum creatinine: a new prediction equation. Modification of Diet in Renal Disease Study Group. Ann Intern Med 1999; 130(6): 461-470.

27. Grading diabetic retinopathy from stereoscopic color fundus photographs--an extension of the modified Airlie House classification. ETDRS report number 10. Early Treatment Diabetic Retinopathy Study Research Group. Ophthalmology 1991; 98(5 Suppl): 786-806.

28. Klein R, Sharrett AR, Klein BE, Moss SE, Folsom AR, Wong TY, et al. The association of atherosclerosis, vascular risk factors, and retinopathy in adults with diabetes : the atherosclerosis risk in communities study. Ophthalmology 2002; 109(7): 1225-1234.

29. Papanas N, Symeonidis G, Mavridis G, Georgiadis GS, Papas TT, Lazarides MK, et al. Ankle-brachial index: a surrogate marker of microvascular complications in type 2 diabetes mellitus? Int Angiol 2007; 26(3): 253-257.

30. Miyamoto M, Kotani K, Okada K, Fujii Y, Konno K, Ishibashi $\mathrm{S}$, et al. The correlation of common carotid arterial diameter with atherosclerosis and diabetic retinopathy in patients with type 2 diabetes mellitus. Acta Diabetol DOI: 10.1007/s00592-011-0287-8.

31. Ogawa Y, Uchigata Y, Iwamoto Y. Progression factors of 
carotid intima-media thickness and plaque in patients with long-term, early-onset type 1 diabetes mellitus in Japan: simultaneous comparison with diabetic retinopathy. $J$ Atheroscler Thromb 2009; 16(6): 821-828.

32. Mostaza JM, Suarez C, Manzano L, Cairols M, LópezFernández F, Aguilar I, et al. Sub-clinical vascular disease in type 2 diabetic subjects: relationship with chronic complications of diabetes and the presence of cardiovascular disease risk factors. Eur J Intern Med 2008; 19(4): 255-260.

33. Klein R, Klein BE, Moss SE, Cruickshanks KJ, Association of ocular disease and mortality in a diabetic population. Arch Ophthalmol 1999; 117(11): 1487-1495. 34. Safar ME, London GM. Therapeutic studies and arterial stiffness in hypertension: recommendations of the European Society of Hypertension. The Clinical Committee of Arterial Structure and Function. Working Group on Vascular Structure and Function of the European Society of Hypertension. J Hypertens 2000; 18(11): $1527-$ 1535.

35. Laurent S, Boutouyrie P, Asmar R, Gautier I, Laloux B, Guize L, et al. Aortic stiffness is an independent predictor of all-cause and cardiovascular mortality in hypertensive patients. Hypertension 2001; 37(5): 1236-1241. 